\title{
УДК 519.17
}

\section{ИСКЛЮЧИТЕЛЬНЫЕ ПСЕВДОГЕОМЕТРИЧЕСКИЕ ГРАФЫ С СОБСТВЕННЫМ ЗНАЧЕНИЕМ $r$}

\begin{abstract}
А. Х. Журтов
А. Ноймайер перечислил параметры сильно регулярных графов с наименьшим собственным значением $-m$. Как следствие, доказано, что для данного натурального числа $r$ существует лишь конечное число псевдогеометрических графов для $p G_{s-r}(s, t)$ с параметрами, отличными от параметров сети $p G_{s-r}(s, s-$ $r$ ) и от параметров $p G_{s-r}(s,(s-r)(r+1) / r)$ ( $s$ делится на $r$ ) дополнительного графа для блочного графа 2-схемы Штейнера. В работе явно указаны такие функции $f(r), g(r)$, что для $s>f(r)$ или для $t>g(r)$ любой псевдогеометрический граф для $p G_{s-r}(s, t)$ имеет параметры сети $p G_{s-r}(s, s-r)$ или параметры $p G_{s-r}(s,(s-r)(r+1) / r)$.
\end{abstract}

Ключевые слова: сильно регулярный граф, псевдогеометрический граф.

A. Kh. Zhurtov. Exceptional pseudogeometric graphs with eigenvalue $r$.

A. Neumaier enumerated the parameters of strongly regular graphs with smallest eigenvalue $-m$. As a corollary it is proved that for a positive integer $r$ there exist only finitely many pseudogeometric graphs for $p G_{s-r}(s, t)$ with parameters different from the parameters of the net $p G_{s-r}(s, s-r)$ and from the parameters of the $p G_{s-r}(s,(s-r)(r+1) / r)$ graph complementary to the line graph of a Steiner 2-design ( $s$ is a multiple of $\left.r\right)$. In this paper we explicitly specify functions $f(r)$ and $g(r)$ such that for $s>f(r)$ or $t>g(r)$ any pseudogeometric graph for $p G_{s-r}(s, t)$ has parameters of the net $p G_{s-r}(s, s-r)$ or parameters of $p G_{s-r}(s,(s-r)(r+1) / r)$.

Keywords: strongly regular graph, pseudogeometric graph.

MSC: $05 \mathrm{C} 25$

DOI: $10.21538 / 0134-4889-2018-24-3-68-72$

Мы рассматриваем неориентированные графы без петель и кратных ребер. Для вершины $a$ графа $\Gamma$ через $\Gamma_{i}(a)$ обозначим $i$-окрестность вершины $a$, т. е. подграф, индуцированный $\Gamma$ на множестве всех вершин, находящихся на расстоянии $i$ от $a$. Подграф $\Gamma(a)=\Gamma_{1}(a)$ называется окрестностъю вершины $a$ и обозначается через $[a]$, если граф $\Gamma$ фиксирован. Положим $a^{\perp}=$ $\{a\} \cup[a]$.

Степенъю вершины называется число вершин в ее окрестности. Граф Г называется регулярным степени $k$, если степень любой вершины $a$ из $\Gamma$ равна $k$. Граф $\Gamma$ назовем реберно регулярным с параметрами $(v, k, \lambda)$, если он содержит $v$ вершин, регулярен степени $k$ и каждое его ребро лежит ровно в $\lambda$ треугольниках. Граф $Г$ - вполне регулярный граф с параметрами $(v, k, \lambda, \mu)$, если он реберно регулярен с соответствующими параметрами и $[a] \cap[b]$ содержит ровно $\mu$ вершин для любых двух вершин $a, b$, находящихся на расстоянии 2 в $Г$. Вполне регулярный граф называется сильно регулярным графом, если он имеет диаметр 2.

Система инцидентности с множеством точек $P$ и множеством прямых $\mathcal{L}$ называется $\alpha$-частичной геометрией порядка $(s, t)$, если каждая прямая содержит ровно $s+1$ точку, каждая точка лежит ровно на $t+1$ прямой, любые две точки лежат не более чем на одной прямой и для любого антифлага $(a, l) \in(P, L)$ найдется точно $\alpha$ прямых, проходящих через $a$ и пересекаюших $l$ (обозначение $p G_{\alpha}(s, t)$ ). В случае $\alpha=1$ геометрия называется обобщенным четырехугольником и обозначается через $G Q(s, t)$, а в случае $\alpha=t$ геометрия называется сетъю. Точечный граф геометрии определяется на множестве точек $P$ и две точки смежны, если они лежат на прямой. Точечный граф геометрии $p G_{\alpha}(s, t)$ сильно регулярен с $v=(s+1)(1+s t / \alpha)$, $k=s(t+1), \lambda=s-1+t(\alpha-1), \mu=\alpha(t+1)$. Сильно регулярный граф с такими параметрами для некоторых натуральных чисел $\alpha, s, t$ называется псевдогеометрическим графом для $p G_{\alpha}(s, t)$. 
А. Ноймайер [1] доказал, что для данного натурального числа $r$ существует лишь конечное число исключительных графов (псевдогеометрических графов для $p G_{s-r}(s, t)$ с параметрами, отличными от параметров сети $p G_{s-r}(s, s-r)$ и от параметров $p G_{s-r}(s,(s-r)(r+1) / r)(s$ делится на $r$ ) дополнительного графа для блочного графа 2-схемы Штейнера).

Мы хотим найти такие функции $f(r), g(r)$, что для $s>f(r)$ или для $t>g(r)$ любой псевдогеометрический граф для $p G_{s-r}(s, t)$ имеет параметры сети $p G_{s-r}(s, s-r)$ или параметры $p G_{s-r}(s,(s-r)(r+1) / r)$.

Теорема. Имеем $g(r)=r(r+1)^{5}-(r+1), f(r)=r+r(r+1) g(r) / 2$.

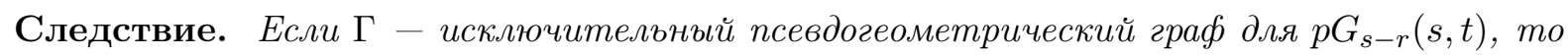
$s \leq r+r(r+1) g(r) / 2, t \leq r(r+1)^{5}-(r+1)$.

Пусть $\Gamma$ - сильно регулярный граф с собственными значениями $n-m,-m$. Из [1, теорема 5.1] следует, что Г является одним из следующих графов:

а) полный многодольный граф с $l$ долями порядка $m$;

б) псевдогеометрический граф для $p G_{m-1}(s, m-1)$;

в) псевдогеометрический граф для $p G_{m}(s, m-1)$;

г) графы из некоторого конечного множества.

Графы из п. а) имеют $n=m, \mu=m(l-1)$ и существуют для любого $l \geq 2$.

Графы из п. б) имеют $\mu=(m-1) m$ и для достаточно большого $s$ являются геометрическими.

Графы из п. в) имеют $\mu=m^{2}$, существуют для $s(s+1)$ кратного $m$, и для достаточно большого $s$ являются геометрическими.

Граф из п. г) имеет множество параметров

$$
(m, n, \mu, \bar{\mu}, f, v, k, \bar{k}, \lambda, \bar{\lambda}), \quad \mu \notin\left\{(m-1) m, m^{2}\right\}, \quad n>m .
$$

Параметры вычисляются по $m, n, \mu$ :

$$
\begin{gathered}
k=\mu+m(n-m), \quad \lambda=\mu+n-2 m, \quad v=1+k+k(k-\lambda-1) / \mu, \\
\bar{k}=v-k-1, \quad \bar{\mu}=v-2 k+\lambda, \quad \bar{\lambda}=v-2 k-2+\mu,
\end{gathered}
$$

кратность собственного значения $n-m$ определяется как $f=(m-1) k(k+m) /(n \mu)$.

Граф назовем исключительным при соблюдении

(1) условия Крейна: $\mu(n-(m-1) m) \leq(m-1)(n-m)(n+(m-1) m)$, если $1<m<n$;

(2) абсолютной границы:

$v \leq f(f+3) / 2(v \leq f(f+1) / 2)$, если $\mu(n-(m-1) m) \neq(m-1)(n-m)(n+(m-1) m) ;$

(3) $\mu$-границы: $\mu \leq m^{3}(2 m-3)$ (в случае равенства имеем $\left.n=m(m-1)(2 m-1)\right)$;

(4) границы для числа 3 -лап: $n \leq m(m-1)(\mu+1) / 2+m-1$.

Лемма 1. Пусть $\bar{\Gamma}-$ псевдогеометрический граф для $p G_{s-r}(s, t)$. Тогда

$$
m=r+1, \quad n=m+t, \quad \bar{b}_{1}=(r+1) t, \quad k=s t(r+1) /(s-r), \quad \mu=r(r+1) t /(s-r) .
$$

В случае $\mu=1$ имеем $t \leq(r+1)^{2} u s \leq r+r(r+1)^{3}$.

Д о к а з а т е л ь с т в о. Так как неглавные собственные значения графа $\bar{\Gamma}$ равны $r$, $-(t+1)$, то $m=r+1, n=m+t=r+t+1$. Далее, $\bar{k}=s(t+1), \bar{\lambda}+1=s+(s-r-1) t$, поэтому $\bar{b}_{1}=(r+1) t$. Имеем $k=(s+1)(1+s t /(s-r)-1-s(t+1)=s t(s /(s-r)+1 /(s-r)-1)=$ $s t(r+1) /(s-r)$. Наконец, $\mu=k-\bar{b}_{1}=r(r+1) t /(s-r)$.

Допустим, что $\mu=1$. Тогда $s=r+r(r+1) t, k=s t(r+1) /(r(r+1) t)=s / r=(r+1) t+1$, $b_{1}=r(t+1)$ и $\lambda=k-b_{1}-1=(r+1) t+1-r(t+1)-1=t-r$. Отсюда $t-r+1$ делит $(r+1) t+1$. 
Далее, $(t-r+1, r t+t+1)=\left(t^{2}-r t+t, r t+t+1\right)=\left((t+1)^{2}, t-r+1\right)$ и для $d=(t+1, r)$ число $t-r+1$ делит $d^{2}$.

По условию целочисленности для $p G_{s-r}(s, t)$ число $r(r+1) t(r+t+1)$ делит $t(t+1)(r+$ $r(r+1))(r+1)^{2}$, поэтому $(r+t+1)$ делит $(t+1)(r+2)(r+1)$. Далее, $(t+r+1, t+1)=(r, t+1)$, $(t+r+1, r+1)=(r+1, t)$ и $(t+r+1, r+2)=(t-1, r+2)$.

В случае $t=r$ имеем $\lambda=0$ и $k \in\{2,3,7,57\}$. Если $k=3$, то $r=t=1$ и $s=3$. Если $k=7$, то $v=50$, поэтому $r=2, t=2$ и $s=14$. Если $k=57$, то $v=3250$, поэтому $r=7, t=7$ и $s=399$. В любом случае ввиду границы Хофмана для коклик графа $\bar{\Gamma}$ имеем $t-r+1 \leq 1+s /(r(r+1))=1+(r t+r+1) /(r+1)$, поэтому $(t-r)(r+1) \leq r t+r+1$ и $t \leq(r+1)^{2}$. Отсюда $s=r+r(r+1) t \leq r+r(r+1)^{3}$.

Лемма 2. ЕслиГ - исключительный граф с неглавными собственными значениями $n-m$, $m$, mo $m<n<m^{5}(m-1) u 1 \leq \mu \leq m^{3}(2 m-3)$.

Д ок аз а т ел ь с т в о. Если $n>m^{4}(m-1)^{2}$, то с учетом неравенства $\mu \leq m^{3} \times$ $(2 m-3)$ имеем $n>m(m-1)(\mu+1) / 2+m-1$ и ввиду границы для числа 3 -лап получаем $\mu \in$ $\left\{(m-1) m, m^{2}\right\}$. Если $n \leq m$, то $n=m$ и $\Gamma$ - полный многодольный граф с $l$ долями порядка $m$. В любом случае имеем противоречие с исключительностью графа. Значит, $m<n<m^{5}(m-1)$ и $1 \leq \mu \leq m^{3}(2 m-3)$.

Лемма 3. Пусть $\Gamma-$ псевдогеометрический граф для $p G_{s-r}(s, t)$. Положим

$$
g(r)=r(r+1)^{5}-(r+1), \quad f(r)=r+r(r+1) g(r) / 2 .
$$

Если $s>f(r)$ или $t>g(r)$, то $\Gamma$ имеет параметры сети $(t=s-r)$ или параметры $p G_{s-r}(s,(s-r)(r+1) / r)$.

Д о к а з а т е л ь с т в о. Пусть $\Gamma-$ псевдогеометрический граф для $p G_{s-r}(s, t)$. По лемме 2 имеем $n=t+r+1<r(r+1)^{5}$. Далее, $\mu=r(r+1) t /(s-r) \geq 1$. В случае $\mu=1$ по лемме 1 имеем $t \leq(r+1)^{2}$ и $s \leq r+r(r+1)^{3}$. В случае $\mu \geq 2$ имеем $s-r \leq r(r+1) t / 2$. Положим $g(r)=r(r+1)^{5}-(r+1), f(r)=r+r(r+1) g(r) / 2$. Тогда заключение леммы выполняется.

Из леммы 3 следует теорема.

Лемма 4. Пусть $\Gamma-$ псевдогеометрический граф для $p G_{\alpha}(s, t)$. Если $s>\alpha$, то $t \leq$ $(s+1-\alpha)^{2}(2 \alpha-1)$.

Д о к а з а т е л ь с т в о. Утверждение доказано Ноймайером [1, теорема 4.5] для геометрического графа. Нетрудно увидеть, что рассуждения верны и для псевдогеометрического графа.

Заметим, что для малых $r$ точные значения функций $f$ и $g$ гораздо меньше. Так, для $r=1$ имеем $f(1)=31$ и $g(1)=30$. С другой стороны, имеются точно два исключительных псевдогеометрических графа с $r=1$ - это точечные графы для $G Q(2,4)$ и $p G_{2}(3,1)$ (треугольный граф $T(5))$. Следовательно, фактические значения $f(1)$ и $g(1)$ равны 3 и 4 соответственно.

Для $r=2$ имеем $f(2)=1451$ и $g(2)=483$. С другой стороны, если $\Gamma-$ исключительный псевдогеометрический граф для $p G_{s-2}(s, t)$, то по [2, теорема 1] параметры $(s, t)$ принимают одно из следующих значений:

(1) $(3,3),(3,5),(3,9),(4,1),(4,7),(4,9),(4,12),(4,17),(4,27)$;

(2) $(5,2),(5,7),(5,9),(5,12),(5,17),(5,27),(6,18),(7,25)$;

(3) $(8,5),(8,15),(8,21),(8,33),(9,42),(10,52)$;

(4) $(14,4),(14,32),(17,65),(20,9),(20,81),(32,5)$.

Следовательно, фактические значения $f(2)$ и $g(2)$ равны 32 и 81 соответственно. 
Для $r=3$ имеем $f(3)=18411$ и $g(3)=3068$. С другой стороны, ввиду [3] если $\Gamma-$ исключительный псевдогеометрический граф для $p G_{s-3}(s, t)$, то параметры $(s, t)$ принимают одно из следующих значений:

(i) $(4,2),(4,4),(4,6),(4,8),(4,11),(4,12),(4,16)$;

(ii) $(5,1),(5,5),(5,6),(5,8),(5,11),(5,14),(5,16),(5,53)$;

(iii) $(6,8),(6,10),(6,17),(6,20),(6,24),(6,38),(6,52)$;

(iv) $(7,8),(7,10),(7,17),(7,20),(7,24),(7,38),(7,52),(7,80),(8,20)$;

(v) $(9,5),(9,11),(9,14),(9,16),(9,26),(9,32),(9,41),(9,56),(9,86),(9,140),(9,220),(9,500)$;

(vi) $(10,52),(10,56),(10,84),(11,32),(11,40),(12,48),(13,35),(14,66)$;

(vii) $(15,2),(15,4),(15,6),(15,8),(15,11),(15,20),(15,26),(15,36),(15,44),(15,56),(15,76)$, $(15,116)$;

(viii) $(17,98),(18,5),(18,110),(20,9),(20,68),(20,136),(21,3),(21,150),(23,65),(23,180)$;

(ix) $(24,56),(25,11),(27,8),(27,104),(31,182),(33,200),(39,6),(39,9),(39,126),(45,14)$, $(45,203)$;

(x) $(55,26),(55,260),(55,416),(63,5),(63,20),(69,341),(105,17),(135,11)$.

Ввиду леммы 4 возможности $(s, t)=(9,220)$ и $(s, t)=(9,500)$ не возникают. Следовательно, фактические значения $f(3)$ и $g(3)$ равны 135 и 416 соответственно.

Для $r=4$ имеем $f(4)=124954, g(4)=12495$.

С другой стороны, ввиду [4] если $Г-$ исключительный псевдогеометрический граф для $p G_{s-4}(s, t)$, то параметры $(s, t)$ принимают одно из следующих значений:

(1) $(5,3),(5,5),(5,7),(5,10),(5,15),(5,19),(5,20),(5,25),(6,1),(6,5),(6,7),(6,9),(6,10)$, $(6,15),(6,16),(6,23),(6,25),(6,30),(6,37),(7,9),(7,15),(7,27),(7,30),(7,51),(7,75)$;

(2) $(8,3),(8,7),(8,10),(8,13),(8,15),(8,19),(8,25),(8,31),(8,35),(8,40),(8,55),(8,67)$, $(8,85),(8,115),(9,4)(9,7),(9,10),(9,13),(9,15),(9,19),(9,25),(9,31),(9,35),(9,40),(9,55)$, $(9,67),(9,85),(9,115),(9,175)$;

(3) $(10,15),(10,39),(10,45),(10,105),(11,28),(11,35),(11,105),(12,34),(12,60),(12,190)$, $(13,99),(13,135),(14,9),(14,15),(14,16),(14,23),(14,25),(14,30),(14,37),(14,55),(14,65)$, $(14,79),(14,100),(14,135),(14,205),(15,55),(15,187),(16,63),(16,75),(16,165)$;

(4) $(18,175),(19,33),(19,75),(19,90),(19,147),(20,100),(21,51),(22,225),(23,133),(24,19)$, $(24,35),(24,45),(24,55),(24,70),(24,95),(24,115),(24,145),(24,195),(24,295),(26,55),(27,115)$, $(27,184),(28,198),(31,243),(32,91),(32,105),(32,259)$;

(5) $(33,435),(34,63),(34,114),(34,135),(34,165),(35,310),(36,328),(38,255),(39,91),(39$, 203), (39, 385), (40,405), (43,468), (44,94), (44,160), (44,490), (47, 559), (48, 275), (48, 583), (49, $135),(49,387),(51,235),(51,658),(52,684),(54,175),(54,325)$;

(6) $(59,231),(64,99),(64,255),(64,315),(64,411),(69,156),(69,455),(74,217),(74,735)$, $(84,352),(90,645),(99,171),(104,275),(104,450),(104,775),(114,869),(114,1375),(119,667)$, $(120,435),(134,598),(139,1107),(144,343),(144,1155), \quad(174,1445), \quad(184,846), \quad(189,1591)$, $(194,1159)$.

Следовательно, фактические значения $f(4)$ и $g(4)$ равны 194 и 1591.

\section{СПИСОК ЛИТЕРАТУРЫ}

1. Neumaier A. Strongly regular graphs with smallest eigenvalue $-m / /$ Arch. Math. 1979. Vol. 33. P. 392-400. doi: 10.1007/BF01222774.

2. Кабанов В.В., Махнев А.А., Падучих Д.В. О сильно регулярных графах с собственным значением 2 и их расширениях // Докл. АН. 2010. Т. 431, № 5. С. 583-586. 
3. Махнев А.А., Падучих Д.В. Исключительные сильно регулярные графы с собственным значением 3 // Докл. АН. 2014. Т. 454, № 1. С. 7-30.

4. Махнев А.А. Сильно регулярные графы с неглавным собственным значением 4 и их расширеня // Изв. Гомельского гос. ун-та. 2014. Т. 84, № 3. С. 84-85.

Журтов Арчил Хазешович

Поступила 05.06.2018

д-р физ.-мат. наук

Кабардино-Балкарский государственный университет им. Х. М. Бербекова,

г. Нальчик

e-mail: zhurtov_a@mail.ru

\section{REFERENCES}

1. Neumaier A. Strongly regular graphs with smallest eigenvalue -m. Arch. Math., 1979, vol. 33, no. 1, pp. 392-400. doi: 10.1007/BF01222774.

2. Kabanov V.V., Makhnev A.A., Paduchikh D.V. On strongly regular graphs with eigenvalue 2 and their extensions. Dokl. Math., 2010, vol. 81, no. 2, pp. 268-271. doi: 10.1134/S1064562410020298.

3. Makhnev A.A., Paduchikh D.V. Exceptional strongly regular graphs with eigenvalue 3. Dokl. Math., 2014, vol. 89, no. 1, pp. 20-23. doi: 10.1134/S1064562414010050.

4. Makhnev A.A. Strongly regular graphs with non-principal eigenvalue 4 and their extensions. Proc. of the F. Scorina Gomel State University, 2014, vol. 84, no. 3, pp. 84-85 (in Russian).

The paper was received by the Editorial Office on July 5, 2018.

Archil Khazeshovich Zhurtov, Dr. Phys.-Math. Sci., Kabardino-Balkarian State University named after H. M. Berbekov, Nal'chik, 360004 Russia, e-mail: zhurtov_a@mail.ru . 